\title{
Electron Dynamics upon Ionization of Polyatomic Molecules: Coupling to Quantum Nuclear Motion and Decoherence
}

\author{
Morgane Vacher \\ Department of Chemistry—Ångström, Uppsala University, Uppsala 75120, Sweden and Department of Chemistry, \\ Imperial College London, London SW7 2AZ, United Kingdom
}

Michael J. Bearpark, Michael A. Robb, and João Pedro Malhado

Department of Chemistry, Imperial College London, London SW7 2AZ, United Kingdom

(Received 29 October 2016; published 23 February 2017)

\begin{abstract}
Knowledge about the electronic motion in molecules is essential for our understanding of chemical reactions and biological processes. The advent of attosecond techniques opens up the possibility to induce electronic motion, observe it in real time, and potentially steer it. A fundamental question remains the factors influencing electronic decoherence and the role played by nuclear motion in this process. Here, we simulate the dynamics upon ionization of the polyatomic molecules paraxylene and modified bismethylene-adamantane, with a quantum mechanical treatment of both electron and nuclear dynamics using the direct dynamics variational multiconfigurational Gaussian method. Our simulations give new important physical insights about the expected decoherence process. We have shown that the decoherence of electron dynamics happens on the time scale of a few femtoseconds, with the interplay of different mechanisms: the dephasing is responsible for the fast decoherence while the nuclear overlap decay may actually help maintain it and is responsible for small revivals.
\end{abstract}

DOI: 10.1103/PhysRevLett.118.083001

Electronic motion initiates specific rearrangements of atoms in molecules that are responsible for chemical reactions and biological processes. Because of the advent of attosecond techniques [1,2], it is possible to induce electron dynamics in molecules. Observing and potentially steering electronic motion on its natural time scale may provide novel pathways towards controlling chemical processes [3-8]. Since the electron distribution is usually considered to be changing much faster than the nuclear geometry, many theoretical studies treat molecular electron dynamics upon ionization as a purely electronic process, at a single static nuclear geometry [9-12]: long-lived oscillatory charge migration is then predicted. The fixed-nuclei and single-geometry approximations have however limited validity [13-19]. The fundamental challenge is to understand to what extent the electronic wave packet retains its coherence, i.e., how long the oscillations in the electronic density survive, in the presence of interactions with the nuclear degrees of freedom.

Using a semiclassical description for the coupled systembath evolution, Fiete and Heller identified three processes that contribute to decoherence of the quantum system [20]: (i) system wave packet displacement, (ii) bath overlap decay, and (iii) phase jitter. In the context of molecular electron

Published by the American Physical Society under the terms of the Creative Commons Attribution 4.0 International license. Further distribution of this work must maintain attribution to the author(s) and the published article's title, journal citation, and DOI. dynamics, the "system" consists of the electrons and the "bath" of the nuclei. The three mechanisms above can respectively be interpreted as (i) change in the electronic state populations, (ii) decrease of the overlap between the nuclear wave packets on different electronic states, and (iii) dephasing of the different wave packet components. The importance of these mechanisms on the coherent electron dynamics upon molecular ionization remains an outstanding question, that we aim to address in the present Letter.

Previous works showed that the nuclear motion treated classically with the Ehrenfest method does not destroy electron dynamics [13-15,19,21-23] but that the intrinsic distribution of geometries in a nuclear wave packet leads to a fast dephasing of the oscillations in the electronic density $[16,18,19]$. The latter prediction was obtained using an ensemble of independent trajectories. However, the effect of the nuclear motion may be underestimated with a mean-field approach [24] and the quantum behavior of the nuclei will not be described by independent trajectories. Also, these methods do not allow the nuclear wave packets on different electronic states to move in different ways; the mechanism (ii) has therefore been completely omitted so far in the theoretical description of electron dynamics upon molecular ionization.

Clearly the treatment of molecular electron dynamics requires a more sophisticated approach with a quantum mechanical treatment of both electron and nuclear dynamics, a great challenge for theory. In this Letter, we use the direct dynamics variational multiconfigurational Gaussian (DDvMCG) method to simulate the effect of quantum nuclear motion on electron dynamics in polyatomic molecules 

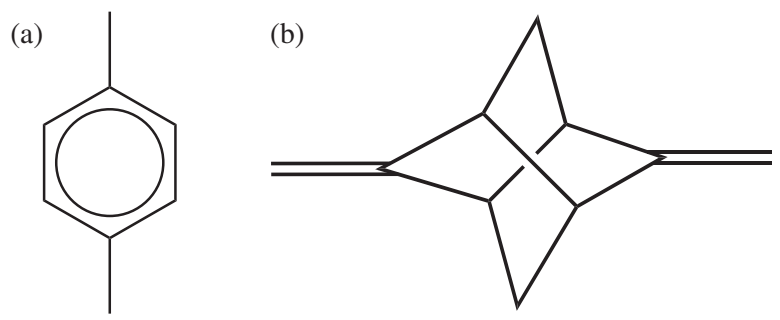

FIG. 1. Structures of (a) paraxylene and (b) BMA[5,5] molecules. BMA $[5,5]$ is a modified bismethylene-adamantane where the cage consists of four connected cyclopentane (instead of cyclohexane) rings [19].

(including all nuclear coordinates). This approach describes the wave packet using a time-dependent basis set of variationally coupled Gaussian functions [23,25,26]. This means a quantum mechanical treatment of both electronic and nuclear degrees of freedom (including their coupling), keeping the advantage of a direct dynamics method [27,28]. We show that for the two molecular systems studied, the nuclear motion leads to decoherence in electron dynamics on the time scale of a few femtoseconds due to the dephasing mechanism, and that, rather counterintuitively, the nuclear overlap decay is actually responsible for small revivals and may help maintain the electronic coherence.

We simulate quantum electron and nuclear dynamics in the following polyatomic molecules studied recently [5,16,19,29,30]: paraxylene and modified bismethyleneadamantane BMA[5,5] cations (Fig. 1). In both molecules, the two lowest-energy electronic states correspond to ionization of the $\pi$ system but the holes created are of different character in paraxylene, and similar in BMA[5,5]. They therefore provide two interesting different cases. The electronic structure is computed using the state-averaged complete active space self-consistent field (CASSCF) method [31] implemented in Gaussian [32]; using the standard 6-31G* basis set, we choose the $\pi$ orbitals as active (6 in para-xylene, 4 in BMA[5,5]). In this Letter, an equal in-phase superposition of the two lowest-energy electronic states is considered. Using the simple picture of a single-fixed nuclear geometry, one predicts long-lived oscillations in the electronic density at a well-defined frequency due to an interference effect between the two electronic states: the hole (or spin) density swings from one side of the phenyl ring to the other side in $5.2 \mathrm{fs}$ in paraxylene (Fig. 2) $[15,16]$ and it migrates from one double bond to the other in BMA[5,5] in 2.4 fs (Fig. 3) [19].

Using the DD-vMCG method (in the single-set formalism) implemented in the Quantics package [33], the quantum nuclear wave packet is represented by a set of coupled Gaussian basis functions (GBF) (with frozen width) $\left\{\chi_{j}\right\}$. The total molecular wave function ansatz reads

$$
\begin{aligned}
\Phi(\mathbf{r}, \mathbf{R}, t) & =\sum_{s} \sum_{j} A_{j}^{(s)}(t) \chi_{j}(\mathbf{R}, t) \phi_{s}(\mathbf{r}) \\
& =\sum_{s} \chi^{(s)}(\mathbf{R}, t) \phi_{s}(\mathbf{r})
\end{aligned}
$$
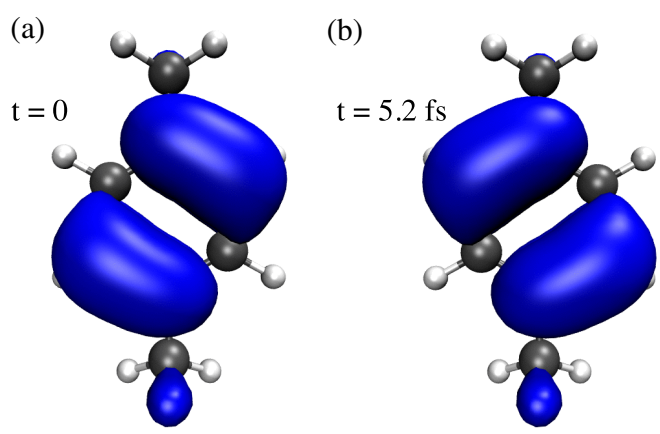

FIG. 2. Electron dynamics in paraxylene cation (at a single fixed nuclear geometry, the equilibrium geometry of the neutral species). (a) The initial electronic wave packet, $1 / \sqrt{2}\left(\phi_{s 1}+\phi_{s 2}\right)$, corresponding to $C=1$ [Eq. (5)], leads to a hole on one side of the phenyl ring. (b) At $t=5.2 \mathrm{fs}$, the time-dependent electronic wave packet becomes $1 / \sqrt{2}\left(\phi_{s 1}-\phi_{s 2}\right)$, corresponding to $C=-1$, and leads to a hole on the other side of the phenyl ring. Figure adapted from Ref. [16].

with $A_{j}^{(s)}$ the coefficient of $\chi_{j}$ on the electronic state $\phi_{s}$, and $\chi^{(s)}(\mathbf{R}, t)=\sum_{j} A_{j}^{(s)}(t) \chi_{j}(\mathbf{R}, t)$ the nuclear wave packet on state $\phi_{s} . \mathbf{r}$ and $\mathbf{R}$ refer to the electronic and nuclear coordinates respectively. The dynamics is run in the basis of diabatic electronic states using the regularization method [34].

Assuming that the system was in its nuclear and electronic ground state before ionization, the simulations are started with the vibrational ground state of the neutral species (in the harmonic approximation). The initial molecular wave function thus reads

$\Phi(\mathbf{r}, \mathbf{R}, t=0)=\frac{1}{\sqrt{2}}\left[\phi_{s_{1}}(\mathbf{r})+\phi_{s_{2}}(\mathbf{r})\right] \chi_{0}(\mathbf{R}, t=0)$.

The time-dependent electronic density is obtained by multiplying the time-dependent molecular wave function

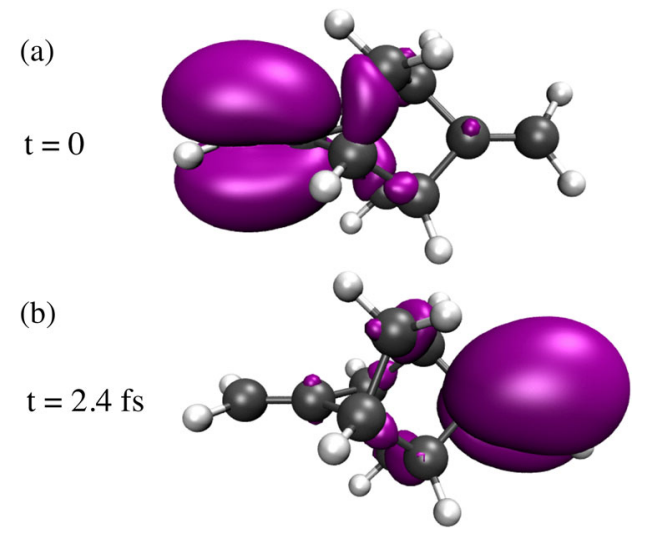

FIG. 3. Same as Fig. 2 in BMA[5,5] cation. (a) The initial electronic wave packet, $1 / \sqrt{2}\left(\phi_{s 1}+\phi_{s 2}\right)$, corresponding to $C=1$ [Eq. (5)], leads to a hole on the left carbon double bond. (b) At $t=2.4 \mathrm{fs}$, the time-dependent electronic wave packet becomes $1 / \sqrt{2}\left(\phi_{s 1}-\phi_{s 2}\right)$, corresponding to $C=-1$, and leads to a hole on the right carbon double bond. Figure adapted from Ref. [19]. 
by its complex conjugate and integrating over all but one electronic spatial coordinate:

$$
\rho(r, \mathbf{R}, t)=\sum_{s^{\prime}} \sum_{s} D^{\left(s s^{\prime}\right)}(\mathbf{R}, t) \rho_{s s^{\prime}}(r),
$$

with the transition density $\rho_{s s^{\prime}}(r)=\int d r^{N-1} \phi_{s}(\mathbf{r}) \phi_{s^{\prime}}(\mathbf{r})$ (for real electronic states) and where the elements of the density operator in the electronic state basis read

$$
\begin{aligned}
D^{\left(s s^{\prime}\right)}(\mathbf{R}, t) & =\left\langle\phi_{s}(\mathbf{r}) \mid \Phi(\mathbf{r}, \mathbf{R}, t)\right\rangle\left\langle\Phi(\mathbf{r}, \mathbf{R}, t) \mid \phi_{s^{\prime}}(\mathbf{r})\right\rangle \\
& =\chi^{(s)}(\mathbf{R}, t) \chi^{\left(s^{\prime}\right) *(\mathbf{R}, t) .}
\end{aligned}
$$

$D_{\text {red }}(t)$ is the reduced density matrix obtained from $D(\mathbf{R}, t)$ by integrating over the nuclear degrees of freedom $\mathbf{R}$. The (complex) off-diagonal elements of the reduced density matrix are called electronic coherences. The (real and positive) diagonal elements of the reduced density matrix give $P^{(s)}$ the populations of the electronic states $\phi_{s}$.

Electron dynamics results in sinusoidal oscillations in the sum of the electronic coherences:

$$
C^{\left(s_{1} s_{2}\right)}(t)=D_{\text {red }}^{\left(s_{1} s_{2}\right)}(t)+D_{\text {red }}^{\left(s_{2} s_{1}\right)}(t) .
$$

Decoherence would lead to a damping of these oscillations. The degree of electronic coherence is measured by $\operatorname{Tr}\left[D_{\text {red }}^{2}(t)\right]$. We can define a quantity $M_{\text {coh }}$ that gathers the relevant terms from $\operatorname{Tr}\left[D_{\text {red }}^{2}(t)\right]$ to quantify electronic coherence [20]:

$$
\begin{aligned}
M_{\mathrm{coh}} & =D_{\text {red }}^{\left(s_{1} s_{2}\right)} D_{\text {red }}^{\left(s_{2} s_{1}\right)}+D_{\text {red }}^{\left(s_{2} s_{1}\right)} D_{\text {red }}^{\left(s_{1} s_{2}\right)} \\
& =2\left|\int d \mathbf{R} \chi^{\left(s_{1}\right)}(\mathbf{R}) \chi^{\left(s_{2}\right) *}(\mathbf{R})\right|^{2} \\
& =2\left|\sum_{l, j} A_{l}^{\left(s_{1}\right)}(t) A_{j}^{\left(s_{2}\right) *}(t) S_{j l}(t)\right|^{2}
\end{aligned}
$$

with $S_{j l}=\left\langle\chi_{j} \mid \chi_{l}\right\rangle$ the overlap between two GBF. Because of the Cauchy-Schwarz inequality,

$$
\begin{aligned}
M_{\text {coh }} & \leq 2 \int d \mathbf{R}\left|\chi^{\left(s_{1}\right)}(\mathbf{R})\right|^{2} \int d \mathbf{R}\left|\chi^{\left(s_{2}\right)}(\mathbf{R})\right|^{2} \\
& =2 P^{\left(s_{1}\right)} P^{\left(s_{2}\right) .}
\end{aligned}
$$

Because of normalization, the coherence is maximal $M_{\mathrm{coh}}=$ $1 / 2$ for equal populations $P^{\left(s_{1}\right)}=P^{\left(s_{2}\right)}=1 / 2$. A complete decoherence would lead to $M_{\text {coh }}=0$. Our aim is to understand the role played by each of the three mechanisms stated above. Mechanism (i) corresponds to the decrease of $M_{\text {coh }}$ from $1 / 2$ to $2 P^{\left(s_{1}\right)} P^{\left(s_{2}\right)}$ due to the transfer of population between electronic states via nonadiabatic relaxation. Then, the Cauchy-Schwarz inequality is strict if $\chi^{\left(s_{1}\right)}(\mathbf{R}, t)$ and $\chi^{\left(s_{2}\right)}(\mathbf{R}, t)$ differ in positions and/or phases. More precisely, mechanism (ii) corresponds to a decrease of the overlaps $S$ while mechanism (iii) corresponds to a dephasing of the different wave packet components.

Let us now start with the electron dynamics results in paraxylene cation [Fig. 4(a)], obtained with 1 GBF for both electronic states (dashed line). In this reference simulation,
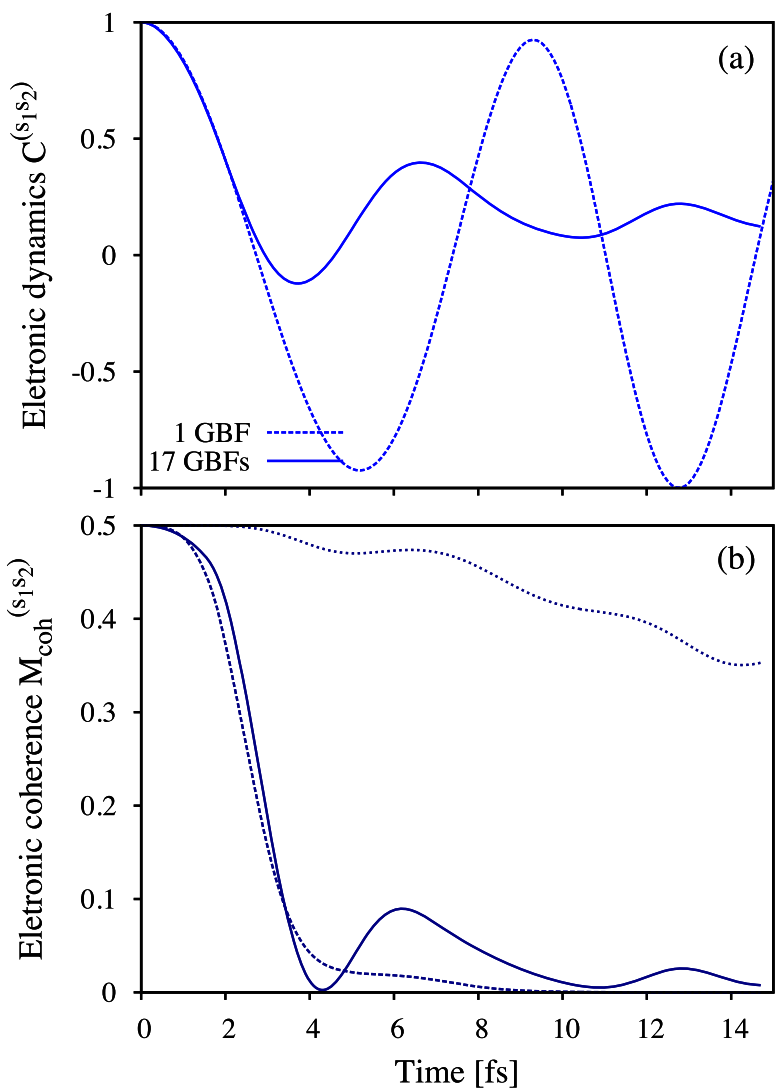

FIG. 4. Electron dynamics coupled with quantum nuclear motion in paraxylene cation. (a) The summed electronic coherence $C^{\left(s_{1} s_{2}\right)}$ in the diabatic basis, in simulations with $1 \mathrm{GBF}$ (dashed line) and 17 GBF (solid line). (b) Degree of electronic coherence with $M_{\text {coh }}$ (solid line), where $S$ is set to 1 (dashed line) and $2 P^{\left(s_{1}\right)} P^{\left(s_{2}\right)}$ (dotted line). The initial electronic wave packet is $1 / \sqrt{2}\left(\phi_{s_{1}}+\phi_{s_{2}}\right)$. The initial nuclear wave packet is the vibrational ground state of the neutral species.

the oscillations in the summed electronic coherence are almost perfect sinusoids: values of around 1 correspond to a hole density as in Fig. 2(a) and values around -1 to a hole density as in Fig. 2(b). Note that the 1-GBF simulation gives a result in agreement with the one obtained with a single Ehrenfest trajectory $[15,23]$. The simulation with $17 \mathrm{GBF}$ [solid line in Fig. 4(a)] shows a more realistic behavior, allowing all three mechanisms to take place. The oscillations are damped very rapidly. After that, the hole does not oscillate back and forth but becomes stationary. $M_{\text {coh }}$ defined in Eq. (6) is plotted in Fig. 4(b) (solid line): there is a very fast electronic decoherence. A value of $M_{\mathrm{coh}}=1 / 4$ is reached at $t \approx 3 \mathrm{fs}$. Interestingly and maybe surprisingly, there are small revivals at $6 \mathrm{fs}$ and $13 \mathrm{fs}$ [35]. To better understand the origin of the observed decoherence (and partial revivals), we have calculated the upper bound of $M_{\mathrm{coh}}$ defined in Eq. (7) (dotted line): it slightly decreases with time because of a slow monotonic increase in $P^{\left(s_{1}\right)}$ (not shown). This shows that mechanism (i) has a small role in the total decoherence. The dashed line represents $M_{\text {coh }}$ with the overlaps $S$ artificially set to 1 . By doing this, we remove 

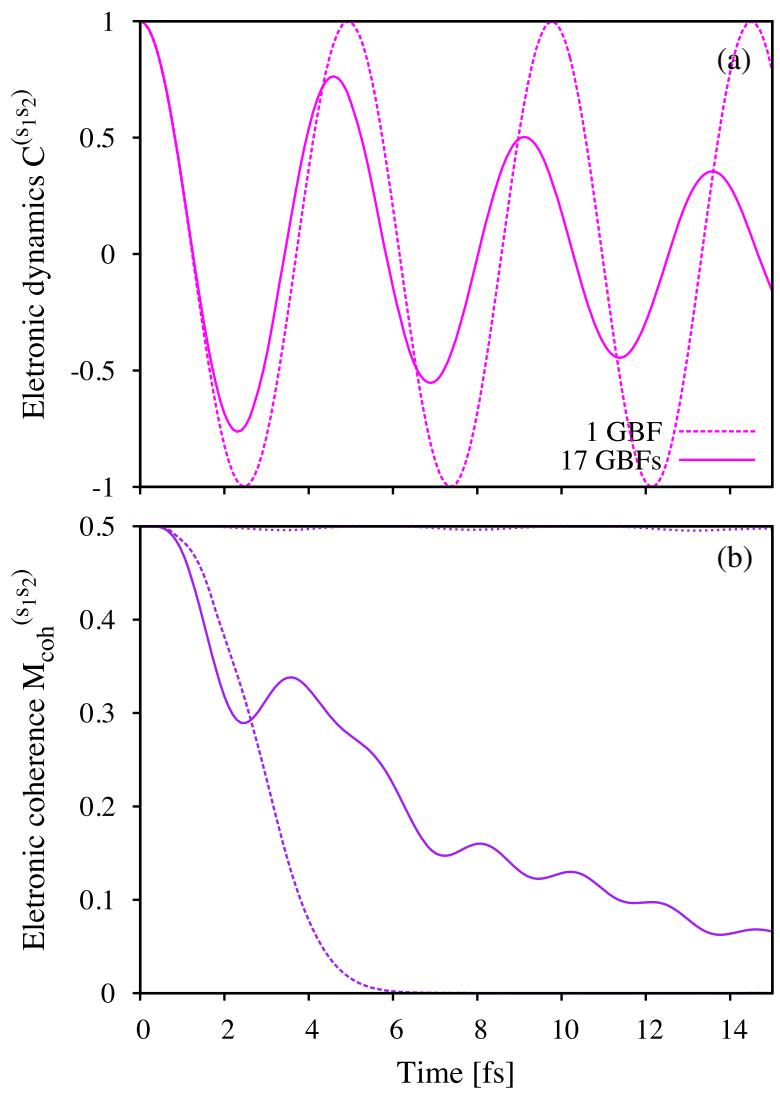

FIG. 5. Same as Fig. 4 in BMA[5,5] cation. The dotted line plotting $2 P^{\left(s_{1}\right)} P^{\left(s_{2}\right)}$ in (b) stays mostly equal to 0.5 .

the contribution of mechanism (ii). The calculated quantity shows a very fast decrease, similar to $M_{\text {coh }}$ (solid line) except for the revivals that have disappeared. This demonstrates that the origin of decoherence is the dephasing mechanism. Note that the dephasing time scale of electron dynamics was assessed previously in paraxylene cation with an ensemble of fixed geometries sampled from the Wigner distribution [16]: the results are in very good agreement.

The electron dynamics results in BMA[5,5] cation are shown in Fig. 5. The summed electronic coherence oscillates-values around 1 correspond to a hole density on the left double bond [Fig. 3(a)] and values around -1 to a hole density on the right double bond [Fig. 3(b)] - in a perfectly sinusoidal manner with $1 \mathrm{GBF}$ [dashed line in Fig. 5(a)]. When considering the more realistic case of $17 \mathrm{GBF}$, the oscillations are again damped with time: electron dynamics decoheres such that the hole stays more delocalized over the two double bonds. There is a fast electronic decoherence, exhibiting interesting structures [solid line in Fig. 5(b)]. A value of $M_{\text {coh }}=1 / 4$ is reached at $t \approx 6 \mathrm{fs}$. The upper bound (dotted line) stays equal to $1 / 2$ showing that mechanism (i) has no effect in the total decoherence. The dashed line shows a very fast and smooth decrease. This indicates that the dephasing mechanism makes a strong contribution to the decoherence process. (This result is in good agreement with the results obtained previously with more approximate methods [19].) The nuclear overlap decay mechanism is responsible for longer and more structured coherence.

Let us end by comparing the two molecular systems studied. Mechanism (i) has little effect in the time scale considered but it is more important in systems like paraxylene where ionization takes place closer to a degeneracy point between the cationic states (due to nonadiabatic relaxation). The dephasing mechanism leads on its own to very fast decoherence, on a similar time scale in both systems ( $\approx 3 \mathrm{fs}$ ). The total decoherence can actually be slower; taking into account the nuclear overlap decay gives rise to small coherence revivals in paraxylene cation and partially compensates for the very fast dephasing in BMA[5,5] cation. Our hypothesis to interpret this somewhat surprising result is the following: The decay of the overlaps $S$ decreases the amplitude of the oscillations for some GBF [Eq. (5)], and thus the dephasing caused by these GBF will be less important, leaving on average a higher amplitude oscillation. We note that a similar effect was observed recently where classical nuclear motion was found to preserve the electronic coherence for slightly longer compared to simulations with nuclei fixed [19]. The fact that the nuclear overlap decay maintains the electronic coherence for longer in BMA[5,5] than in paraxylene is a consequence of different nuclear motions in the two systems. In paraxylene, the two states have different characters, the difference in slopes between the two surfaces is large, and the two nuclear wave packets move far away from each other. In BMA[5,5], the two states have similar character, the difference in slopes is 5 times smaller than in paraxylene and thus, the nuclear wave packets move only slightly away, limiting the effect of dephasing but still maintaining some significant overlap.

To conclude, in this Letter we have presented simulations of the dynamics upon ionization of the polyatomic molecules paraxylene and BMA[5,5], with a quantum mechanical treatment of both electron and nuclear dynamics. Using the DD-vMCG method, we give a new more realistic description of molecular electron dynamics (including all nuclear coordinates) that goes far beyond current approaches to electron dynamics in polyatomic molecular systems (which rely on either the fixed-nuclei approximation or the Ehrenfest approximation). In our simulations, all three (de) coherence mechanisms can potentially take place; in particular, the nuclear wave packets on the different electronic states have the possibility to move in different directions.

Our quantum simulations predict a fast electronic decoherence on a few femtosecond time scale, suppressing the long-lived oscillations in the electronic density. Our simulations also allow deeper insights into the decoherence mechanism. The fast decoherence of electron dynamics seems to be due to the dephasing mechanism while the nuclear overlap decay is actually responsible for small revivals. From the two systems studied here, we have identified qualitative criteria that may be important for the efficiency and time scale of the decoherence. The proximity to a degeneracy point in the cationic states determines the nonadiabatic relaxation and 
therefore the decoherence via mechanism (i). The similarity in character of the states involved determines, via the difference in slopes of the corresponding potential energy surfaces, the relative motion of the two nuclear wave packets: similar states and small relative motion will maintain coherence for longer via mechanism (ii).

To observe electron dynamics in experiments, several measurements have already been proposed: time-resolved Auger spectra [36], photoelectron angular distributions [10], x-ray absorption [37,38], and X-ray raman [39] spectra. Such experiments would allow the observation of the decoherence of electron dynamics that we predict and thereby test the limits of the current theoretical understanding. Although the dynamics studied here result from a linear combination of two electronic states, the methodology employed can be applied to an arbitrary discrete superposition of states created by ionization. It would be interesting in the future to investigate the decoherence mechanisms for electronic states of different energy ranges within the same molecule. The next challenge for theory is to design "good" molecular systems where oscillations in the electronic density persist for longer times.

This work was supported by UK-EPSRC Grant No. EP/ I032517/1. All calculations were run using the Imperial College High Performance Computing service.

[1] M. Hentschel, R. Kienberger, C. Spielmann, G. A. Reider, N. Milosevic, T. Brabec, P. Corkum, U. Heinzmann, M. Drescher, and F. Krausz, Nature (London) 414, 509 (2001).

[2] P. M. Paul, E. S. Toma, P. Breger, G. Mullot, F. Augé, P. Balcou, H. G. Muller, and P. Agostini, Science 292, 1689 (2001).

[3] F. Krausz and M. Ivanov, Rev. Mod. Phys. 81, 163 (2009).

[4] G. Sansone, F. Kelkensberg, J. F. Perez-Torres, F. Morales, M. F. Kling, W. Siu, O. Ghafur, P. Johnsson, M. Swoboda, E. Benedetti, F. Ferrari, F. Lepine, J. L. Sanz-Vicario, S. Zherebtsov, I. Znakovskaya, A. L'Huillier, M. Y. Ivanov, M. Nisoli, F. Martin, and M. J. J. Vrakking, Nature (London) 465, 763 (2010).

[5] F. Calegari, D. Ayuso, A. Trabattoni, L. Belshaw, S. De Camillis, S. Anumula, F. Frassetto, L. Poletto, A. Palacios, P. Decleva, J. B. Greenwood, F. Martín, and M. Nisoli, Science 346, 336 (2014).

[6] L. Fang, T. Osipov, B. F. Murphy, A. Rudenko, D. Rolles, V. S. Petrovic, C. Bostedt, J. D. Bozek, P. H. Bucksbaum, and N. Berrah, J. Phys. B 47, 124006 (2014).

[7] F. Lepine, M. Y. Ivanov, and M. J. J. Vrakking, Nat. Photonics 8, 195 (2014).

[8] S. R. Leone, C. W. McCurdy, J. Burgdorfer, L. S. Cederbaum, Z. Chang, N. Dudovich, J. Feist, C. H. Greene, M. Ivanov, R. Kienberger, U. Keller, M. F. Kling, Z.-H. Loh, T. Pfeifer, A. N. Pfeiffer, R. Santra, K. Schafer, A. Stolow, U. Thumm, and M. J. J. Vrakking, Nat. Photonics 8, 162 (2014).

[9] J. Breidbach and L. S. Cederbaum, J. Chem. Phys. 118, 3983 (2003).

[10] B. Mignolet, R. D. Levine, and F. Remacle, J. Phys. B 47, 124011 (2014).
[11] A. I. Kuleff and L. S. Cederbaum, J. Phys. B 47, 124002 (2014).

[12] A. I. Kuleff, N. V. Kryzhevoi, M. Pernpointner, and L. S. Cederbaum, Phys. Rev. Lett. 117, 093002 (2016).

[13] D. Mendive-Tapia, M. Vacher, M. J. Bearpark, and M. A. Robb, J. Chem. Phys. 139, 044110 (2013).

[14] M. Vacher, M. J. Bearpark, and M. A. Robb, J. Chem. Phys. 140, 201102 (2014).

[15] M. Vacher, D. Mendive-Tapia, M. J. Bearpark, and M. A. Robb, J. Chem. Phys. 142, 094105 (2015).

[16] M. Vacher, L. Steinberg, A. J. Jenkins, M. J. Bearpark, and M. A. Robb, Phys. Rev. A 92, 040502 (2015).

[17] Z. Li, O. Vendrell, and R. Santra, Phys. Rev. Lett. 115, 143002 (2015).

[18] A. J. Jenkins, M. Vacher, M. J. Bearpark, and M. A. Robb, J. Chem. Phys. 144, 104110 (2016).

[19] M. Vacher, F. E. A. Albertani, A. J. Jenkins, I. Polyak, M. J. Bearpark, and M. A. Robb, Faraday Discuss. 194, 95 (2016).

[20] G. A. Fiete and E. J. Heller, Phys. Rev. A 68, 022112 (2003).

[21] M. Lara-Astiaso, D. Ayuso, I. Tavernelli, P. Decleva, A. Palacios, and F. Martin, Faraday Discuss. 194, 41 (2016).

[22] M. Vacher, D. Mendive-Tapia, M. J. Bearpark, and M. A. Robb, Theor. Chem. Acc. 133, 1505 (2014).

[23] M. Vacher, M. J. Bearpark, and M. A. Robb, Theor. Chem. Acc. 135, 187 (2016).

[24] I. S. Ulusoy and M. Nest, J. Phys. Chem. A 116, 11107 (2012).

[25] I. Burghardt, H.-D. Meyer, and L. S. Cederbaum, J. Chem. Phys. 111, 2927 (1999).

[26] G. A. Worth and I. Burghardt, Chem. Phys. Lett. 368, 502 (2003).

[27] G. A. Worth, M. A. Robb, and B. Lasorne, Mol. Phys. 106, 2077 (2008).

[28] G. W. Richings, I. Polyak, K. E. Spinlove, G. A. Worth, I. Burghardt, and B. Lasorne, Int. Rev. Phys. Chem. 34, 269 (2015).

[29] V. Despré, A. Marciniak, V. Loriot, M. C. E. Galbraith, A. Rouzée, M. J. J. Vrakking, F. Lépine, and A. I. Kuleff, J. Phys. Chem. Lett. 6, 426 (2015).

[30] D. R. Austin, F. McGrath, L. Miseikis, D. Wood, P. Hawkins, A. S. Johnson, M. Vacher, Z. Masin, A. Harvey, M. Ivanov, O. Smirnova, and J. P. Marangos, Faraday Discuss. 194, 349 (2016).

[31] B. O. Roos, P. R. Taylor, and P. E. Siegbahn, Chem. Phys. 48, 157 (1980).

[32] M. J. Frisch et al., Gaussian Development Version, Revision h.32 (Gaussian, Inc., Wallingford, 2010).

[33] G. A. Worth, H.-D. Meyer, M. H. Beck, I. Burghardt, A. Jäckle, K. Giri, B. Lasorne, F. Otto, G. W. Richings, and M. Schröder, Quantics: A Computer Package for Quantum Dynamics, Development Version 1.0 (University of Birmingham, Birmingham, 2015).

[34] H. Köppel and B. Schubert, Mol. Phys. 104, 1069 (2006).

[35] L. S. Cederbaum, J. Chem. Phys. 128, 124101 (2008).

[36] B. Cooper and V. Averbukh, Phys. Rev. Lett. 111, 083004 (2013).

[37] A. D. Dutoi, K. Gokhberg, and L. S. Cederbaum, Phys. Rev. A 88, 013419 (2013).

[38] A. D. Dutoi and L. S. Cederbaum, Phys. Rev. A 90, 023414 (2014).

[39] M. Kowalewski, K. Bennett, K. E. Dorfman, and S. Mukamel, Phys. Rev. Lett. 115, 193003 (2015). 